\title{
A unified molecular mechanism for the regulation of acetyl-CoA carboxylase by phosphorylation
}

\author{
Jia Wei ${ }^{1}$, Yixiao Zhang ${ }^{2}$, Tai-Yuan Y $^{3}$, Kianoush Sadre-Bazzaz ${ }^{1}$, Michael J Rudolph ${ }^{1}$, Gabriele A Amodeo ${ }^{1}$, \\ Lorraine S Symington ${ }^{3}$, Thomas Walz ${ }^{2}$, Liang Tong ${ }^{1}$ \\ ${ }^{1}$ Department of Biological Sciences, Columbia University, New York, NY, USA, ${ }^{2}$ Laboratory of Molecular Electron Microscopy, \\ Rockefeller University, New York, NY, USA, ${ }^{3}$ Department of Microbiology and Immunology, Columbia University, Medical \\ Center, New York, NY, USA
}

Acetyl-CoA carboxylases (ACCs) are crucial metabolic enzymes and attractive targets for drug discovery. Eukaryotic acetyl-CoA carboxylases are $250 \mathrm{kDa}$ single-chain, multi-domain enzymes and function as dimers and higher oligomers. Their catalytic activity is tightly regulated by phosphorylation and other means. Here we show that yeast ACC is directly phosphorylated by the protein kinase SNF1 at residue Ser1157, which potently inhibits the enzyme. Crystal structure of three ACC central domains (AC3-AC5) shows that the phosphorylated Ser1157 is recognized by Arg1173, Arg1260, Tyr1113 and Ser1159. The R1173A/R1260A double mutant is insensitive to SNF1, confirming that this binding site is crucial for regulation. Electron microscopic studies reveal dramatic conformational changes in the holoenzyme upon phosphorylation, likely owing to the dissociation of the biotin carboxylase domain dimer. The observations support a unified molecular mechanism for the regulation of ACC by phosphorylation as well as by the natural product soraphen A, a potent inhibitor of eukaryotic ACC. These molecular insights enhance our understanding of acetyl-CoA carboxylase regulation and provide a basis for drug discovery.

Keywords: fatty acid metabolism; metabolic syndrome; enzyme regulation; enzyme phosphorylation

Cell Discovery (2016) 2, 16044; doi:10.1038/celldisc.2016.44; published online 29 November 2016

\section{Introduction}

Acetyl-CoA carboxylases (ACCs) have crucial roles in fatty acid biosynthesis and oxidation and are promising targets for drug discovery against diabetes, cancer and other diseases [1-6]. ACC carries two distinct catalytic activities, biotin carboxylase (BC) and carboxyltransferase (CT), and its biotin is linked covalently to the biotin carboxyl carrier protein (BCCP). Although bacterial ACCs contain multiple subunits that support these different functions, most eukaryotic ACCs are single-chain, multidomain enzymes with a molecular weight of $\sim 250 \mathrm{kDa}$ (Figure 1a) and are active as dimers and higher oligomers.

Correspondence: Liang Tong

Tel: +1 2128545203

E-mail: 1tong@columbia.edu

Received 30 September 2016; accepted 24 October 2016
We recently reported the crystal structure of fulllength yeast ACC (ScACC) [7] (Figure 1b), revealing the overall architecture of this $500 \mathrm{kDa}$ holoenzyme dimer. The BC and CT domain dimers are located at the top and bottom of the structure, respectively. The unique central region of ACC (Supplementary Figure S1), with five domains (AC1-AC5, AC: ACC Central, Figure 1a), is located at the sides, far away from the two active sites. It likely acts as a scaffold to position the $\mathrm{BC}$ and $\mathrm{CT}$ dimers correctly for catalysis. $\mathrm{BCCP}$ is located in the CT active site. The structures of the $\mathrm{BC}, \mathrm{CT}$ and $\mathrm{BCCP}$ domains alone and those of other biotin-dependent carboxylase holoenzymes have also been reported [1, 8-14].

The catalytic activity of eukaryotic ACCs is tightly regulated by phosphorylation, ligand binding and other means. They are potently inhibited upon phosphorylation by AMP-activated protein kinase (AMPK; known as SNF1 in yeast) [15-17]. The sites of AMPK phosphorylation in human ACC1 include Ser80 (Figure 1c) and Ser1216 (Figure 1d). Residue Ser80 is 


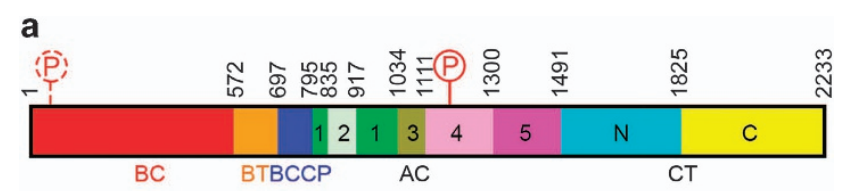

b

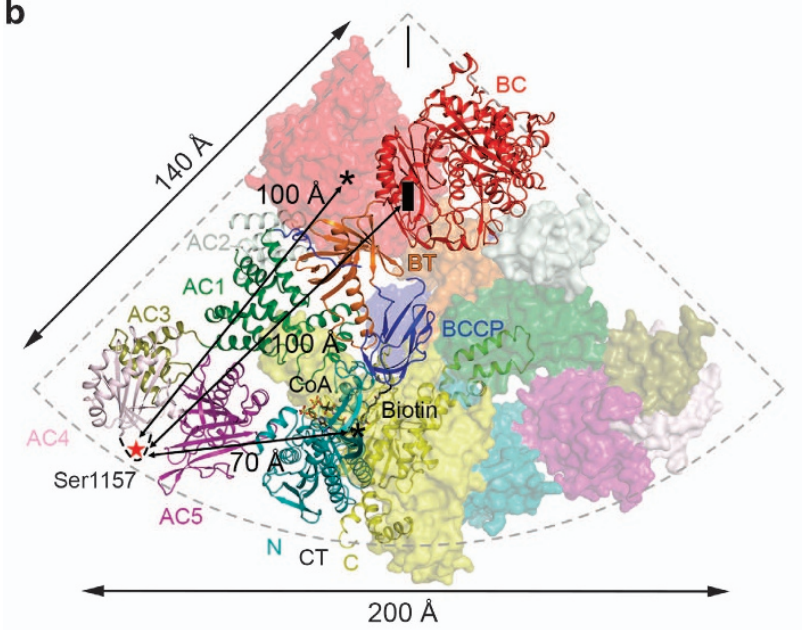

C $\begin{array}{lc}\text { ScACC } & \text { 28-LPGHFIGLNT-37 } \\ \text { RnACC1 } & \text { 74-MRSSMSGLHL-83 } \\ \text { HsACC1 } & \text { 75-IRSSMSGLHL-84 } \\ \text { HsACC2 } & 217-\text { MRPSMSGLHL-226 }\end{array}$

Figure 1 Overall structure of yeast acetyl-CoA carboxylase (ACC) (ScACC). (a) Domain organization of ScACC. The domains are labeled and given different colors. The five domains of ACC Central (AC1-AC5) are labeled 1-5. The phosphorylation site in the central region is indicated. The phosphorylation site before the biotin carboxylase $(B C)$ domain core is indicated with the dashed lines, as it is absent in ScACC. (b) Structure of the ScACC holoenzyme dimer [7]. One protomer is shown as ribbons, while the other as a surface. The domains in the monomers are colored according to panel (a) and labeled. Ser1157 (red star) is located in a loop missing in the structure (dashed lines), and its distances to the $\mathrm{BC}$ and carboxyltransferase (CT) active sites (black asterisks) and the $\mathrm{BC}$ dimer interface (black rectangle) in the holoenzyme are indicated. (c) Sequence conservation near the phosphorylation site before the $\mathrm{BC}$ domain core in animal ACCs. Sc: Saccharomyces cerevisiae, Rn: Rattus novegicus, Hs: Homo sapiens. (d) Sequence conservation near the phosphorylation site in the central region. The structure figures were produced with PyMOL (www.pymol.org).

located prior to the $\mathrm{BC}$ domain core, which starts at residue 101 . The segment containing phosphorylated Ser80 is recognized by a pocket [18] formed through a large conformational change at the $\mathrm{BC}$ dimer interface [7], which makes the $\mathrm{BC}$ domain incompatible with dimerization (Supplementary Figure S2). Monomeric $\mathrm{BC}$ domain also has a conformational change in the active site region, which would block biotin binding. Therefore, phosphorylation of Ser80 stabilizes a conformation of the holoenzyme in which the BC domain dimer is dissociated, and this monomeric state of the $\mathrm{BC}$ domain is catalytically inactive [7]. This new pocket in the dimer interface is also used by soraphen $\mathrm{A}$ [19], a polyketide natural product that potently inhibits eukaryotic ACCs [1], suggesting that it may have a similar mechanism of action as Ser80 phosphorylation (Supplementary Figure S2).

In contrast, ScACC does not have a phosphorylation site equivalent to Ser80 in human ACC1 (Figure 1c). Proteomic studies have identified a large number of phosphorylation sites in ScACC [20, 21]. Among these, the segment around Ser1157 appears to be well conserved with the Ser1216 phosphorylation site in human ACC1 (Figure 1d). Ser1157 is located in a loop containing residues 1137-1170 in domain AC4 (Figure 1a, Supplementary Figure S1), and most of the residues in this loop are disordered in the unphosphorylated ScACC holoenzyme structure, although weak electron density was observed for residues 1153-1161 in one of the molecules [7]. This site is $\sim 70 \AA$ from the nearest CT active site and $\sim 100 \AA$ from the nearest $\mathrm{BC}$ active site in that structure (Figure $1 \mathrm{~b}$ ).

It has been shown that mutating Ser1157 to alanine leads to increased catalytic activity under conditions where SNF1 is activated [22, 23]. Most recently, the crystal structure of phosphorylated AC1-AC5 of ScACC was reported, with Ser1157 having been phosphorylated during expression in insect cells [24]. The binding mode of phosphorylated Ser1157 was defined and the dynamic behavior of ACC holoenzymes was characterized.

However, currently there are no experimental data showing that SNF1 can directly phosphorylate Ser1157 and whether there are additional sites of SNF1 phosphorylation in ScACC. Here we have developed an in vitro phosphorylation system and shown that SNF1 can directly phosphorylate ScACC at Ser1157. We have determined the crystal structure at $2.9 \AA$ resolution of phosphorylated AC3-AC5 domains alone and identified solution conditions that allowed us to directly visualize the ScACC conformation that is observed in the crystal by electron microscopy (EM). EM studies on phosphorylated ScACC reveal dramatic conformational changes, likely owing to dissociation of the $\mathrm{BC}$ domain dimer in the holoenzyme. Based on these observations, we propose a unified molecular mechanism for how AMPK phosphorylation (at both sites in animal ACCs) and soraphen A binding regulate the catalytic activity of eukaryotic ACCs. 


\section{Results}

Ser1157 is directly phosphorylated by SNF1

To obtain experimental evidence for the direct phosphorylation of Ser1157 by SNF1, we carried out in vitro phosphorylation reactions and monitored their progress by ACC activity assays and/or sodium dodecyl sulfate polyacrylamide gels, noting that a phosphorylated protein generally runs slower than its unphosphorylated counterpart. We overexpressed and purified a SNF1 heterotrimer in Escherichia coli, containing the Snf1 catalytic subunit and the Gal83 and Snf4 regulatory subunits, using the same method as that for expressing the SNF1 heterotrimer core (missing primarily the protein kinase domain) [25]. To activate SNF1, we expressed and purified the constitutively active upstream protein kinase Tos3 [26]

a
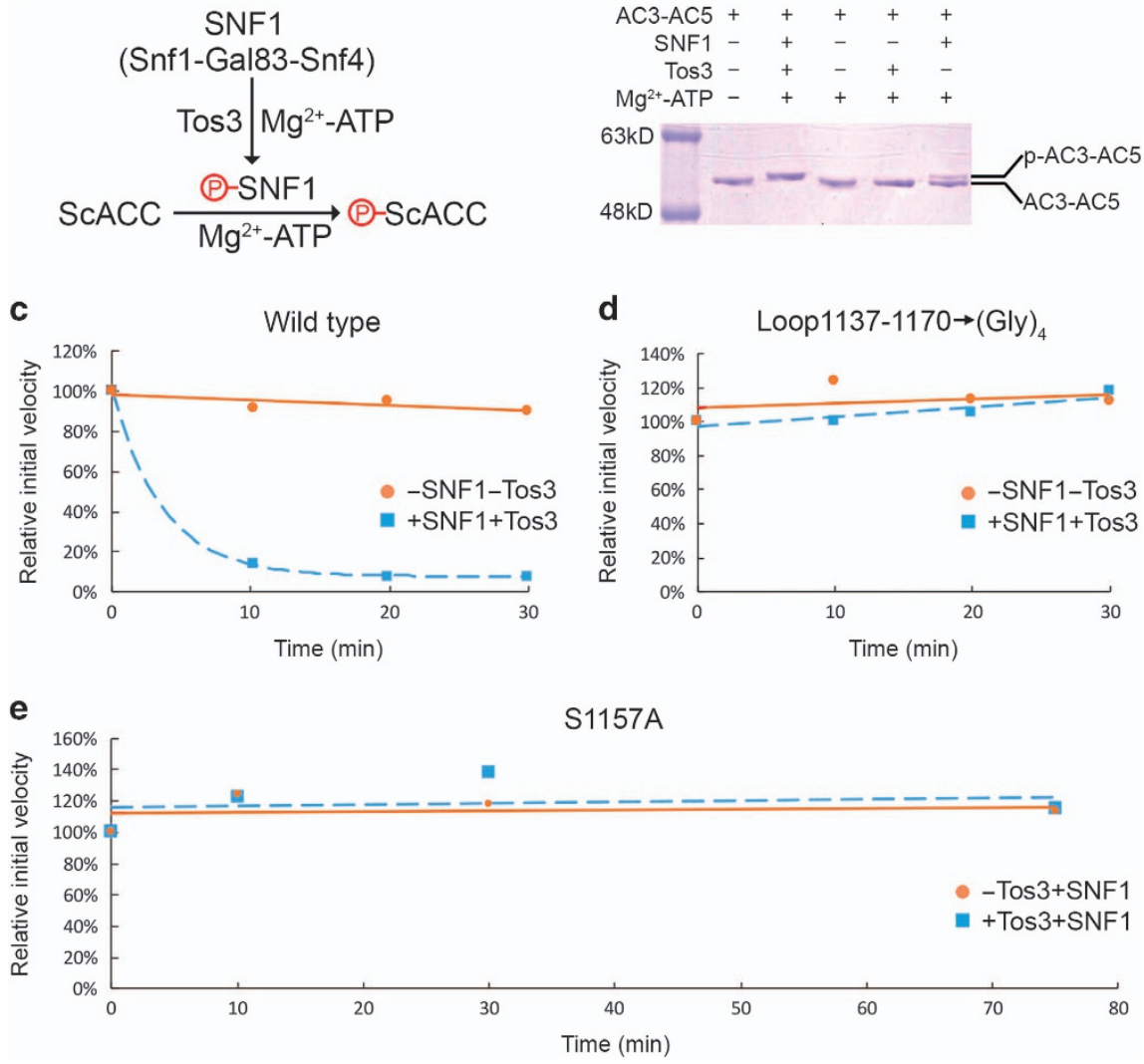
presence of ATP and $\mathrm{Mg}^{2+}$ (Figure 2a). produce any phosphorylated AC3-AC5.

b

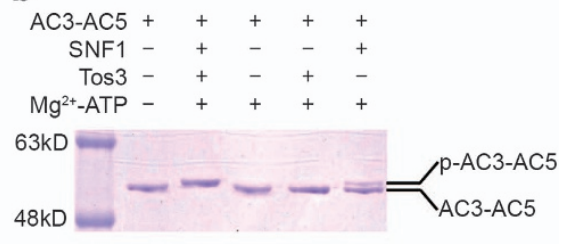

d Loop1137-1170 $\rightarrow(\text { Gly })_{4}$

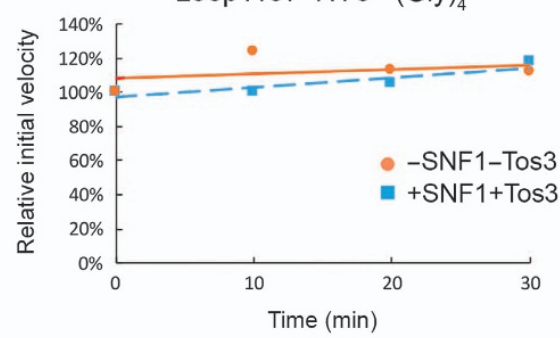

S1157A

in $E$. coli. We were then able to produce phosphorylated ScACC by incubation with SNF1 and Tos3 in the

We observed a clear shift in the position in sodium dodecyl sulfate gel for domains AC3-AC5 after treatment with Tos3 and SNF1 but not in their absence (Figure 2b), confirming that SNF1 can directly and completely phosphorylate this segment of ScACC (most likely on Ser1157) under the reaction condition tested. Interestingly, SNF1 alone (without Tos3) could also produce a small amount of phosphorylated AC3-AC5, suggesting that it could be weakly active in this buffer. In comparison, Tos 3 alone could not

For full-length ScACC, we used activity assays to monitor the phosphorylation because a gel shift was difficult to visualize owing to its large size. We 
observed rapid loss of ScACC activity upon incubation with SNF1 and Tos3, with $\sim 80 \%$ of the activity being lost within $10 \mathrm{~min}$ (Figure 2c). In comparison, no activity loss was observed, even after $30 \mathrm{~min}$ incubation, for an ACC mutant in which residues 1137-1170 were replaced with a $(\mathrm{Gly})_{4}$ linker (Figure 2d). Most importantly, SNF1 had no effect on the activity of the S1157A mutant, even after prolonged incubation (Figure 2e). This result indicates that Ser1157 is the predominant if not the sole SNF1 phosphorylation site in ScACC that is capable of regulating its catalysis.

\section{Crystal structure of phosphorylated AC3-AC5}

To illuminate the molecular basis for how phosphorylated Ser1157 (pSer1157) is recognized by ScACC, we determined the crystal structure at $2.9 \AA$ resolution of domains AC3-AC5 with Ser1157 fully phosphorylated with our in vitro phosphorylation system (Figure 3a, Table 1). The overall structures of the two AC3-AC5 molecules in the asymmetric unit are similar, with root mean square (r.m.s.) distance of $0.45 \AA$ for 402 equivalent $\mathrm{C} \alpha$ atoms between them (Supplementary Figure S3). pSer1157 is observed in both molecules and has essentially the same conformation, but the loop containing this residue is more ordered in one of the two molecules (residues 1137-1143 and 1156-1169 are modeled in one molecule while only residues $1156-1162$ are modeled in the other; Supplementary Figure S3) and that molecule will be described further below.

The loop containing pSer1157 is located in a groove at the interface between domains AC4 and AC5, with pSer1157 positioned in an electropositive pocket in domain AC4 (Figure 3b). The phosphate group interacts with the side chains of Arg1173, Arg1260, Tyr1113 and Ser1159 (Figure 3c), and these residues are well conserved among those eukaryotic ACCs that contain this phosphorylation site (Supplementary Figure S1).

To assess the functional importance of this binding site, we created the R1173A/R1260A double mutant and found that its catalytic activity was only mildly inhibited upon treatment with activated SNF1 (Figure 3d). This confirms the structural observations and indicates that this binding site is crucial for the regulation of yeast ACC by SNF1.

The overall structure of the phosphorylated AC3-AC5 is nearly the same as that of unphosphorylated AC3-AC5 alone, with r.m.s. distance of $0.48 \AA$ for their 402 equivalent $\mathrm{C} \alpha$ atoms (Figure 3a). However, there is a large difference in the position of $\mathrm{AC} 5$ relative to $\mathrm{AC} 3-\mathrm{AC} 4$ compared with the structure of these domains in the holoenzyme [7]. With domains
AC3-AC4 in overlay between the structures of the (phosphorylated) AC3-AC5 and the holoenzyme, the orientation of AC5 differs by a rotation of $40^{\circ}$ [7] (Figure 3e). Moreover, unphosphorylated Ser1157 in the holoenzyme structure is located in a different pocket, at the AC4-AC5 interface and $\sim 16 \AA$ away from pSer1157 (Figure 3f), suggesting a large conformational change for the loop containing Ser1157 upon its phosphorylation.

The overall structure of phosphorylated AC3-AC5 is similar to that of phosphorylated AC1-AC5 of ScACC reported recently [24], with r.m.s. distance of $0.63 \AA$ for 391 equivalent $\mathrm{C} \alpha$ atoms between them (Supplementary Figure S4). The binding modes of pSer1157 in the two structures are similar as well (Supplementary Figure S4). However, there are conformational differences between the two structures for the rest of this loop, especially residues 1140-1143, which have well-defined electron density (Supplementary Figure S4). Phe1140 is in contact with Phe1298 in one structure while Phe1143 is in contact with Phe1298 in the other (Supplementary Figure S4).

\section{Conformational variability for the ACC holoenzyme}

To reveal how phosphorylation at Ser1157 inhibits the activity of ACC, we attempted to determine the crystal structure of phosphorylated full-length ScACC but were not able to obtain diffraction-quality crystals after extensive efforts. We then turned to EM.

The crystal structure of the ScACC holoenzyme shows that it adopts the shape of a quarter of a disk (Figure 1b) [7]. To our surprise, when we examined this sample in the regular protein buffer $(20 \mathrm{~mm}$ Tris (pH 7.5) and $300 \mathrm{~mm} \mathrm{NaCl}$ ) by negative-stain EM, we observed primarily elongated shapes, varying from completely straight to bent, with only a few particles having a compact shape similar to that seen in the crystal (Figure 4a). The elongated shapes are likely caused by the dissociation of the $\mathrm{BC}$ domain dimer, and such conformations of the holoenzyme are probably catalytically inactive.

Noting that we were able to observe the compact structure in the crystal, we hypothesized that the crystallization condition may have stabilized that conformation of the ScACC holoenzyme. Consistent with our hypothesis, mostly compact shapes were observed when we prepared negative-stain EM grids with ScACC in the reservoir solution used for crystallization (data not shown). The solution contained 14\% (w/v) PEG3350, 4\% (v/v) tert-butanol and $0.2 \mathrm{M}$ sodium citrate [7]. Further testing showed that citrate alone from this solution was sufficient to produce the 
a

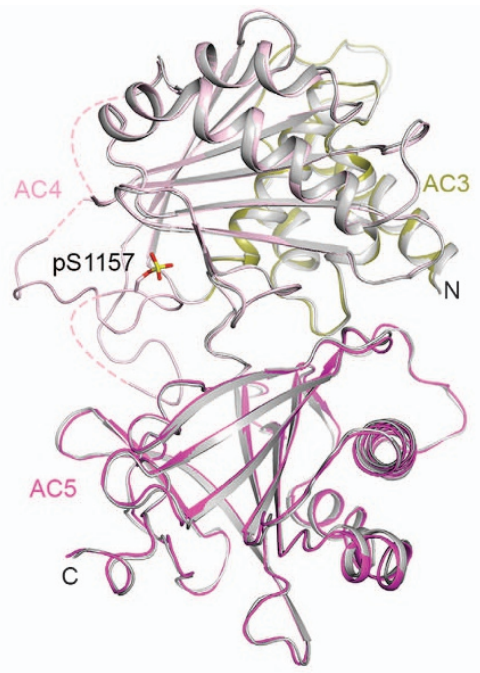

C
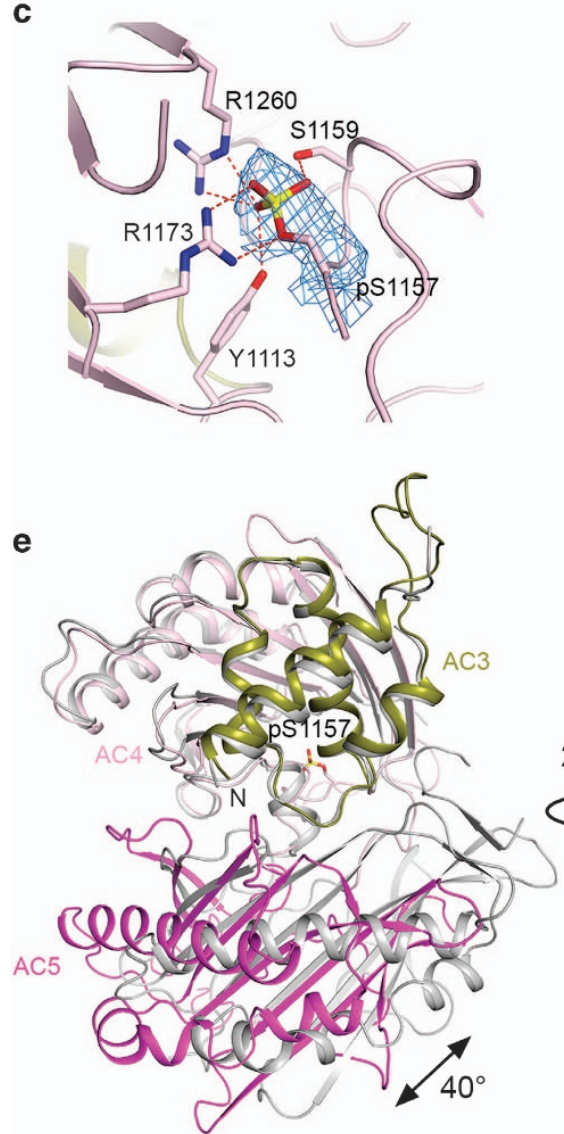

b

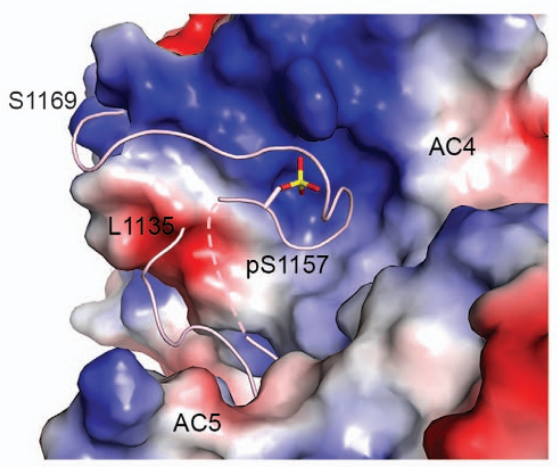

d

R1173A/R1260A

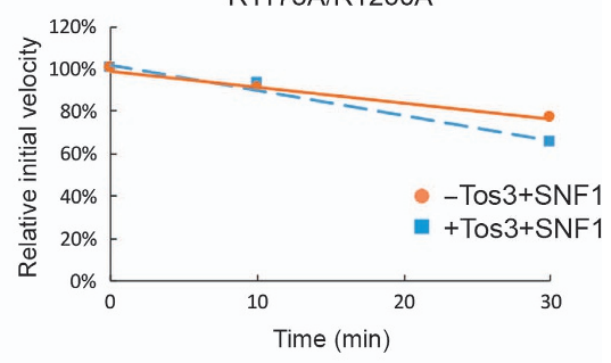

f

Figure 3 Crystal structure of phosphorylated domains AC3-AC5 of yeast acetyl-CoA carboxylase (ScACC). (a) Overlay of the structure of phosphorylated AC3-AC5 (in color) with that of unphosphorylated AC3-AC5 alone (gray). The pSer1157 side chain is shown as stick models. (b) Molecular surface of ScACC near the loop containing the pSer1157 residue, colored by electrostatic potential (blue: positive; red: negative). (c) The binding site for pSer1157 in domain AC4. Interactions with the phosphate are indicated with dashed lines (red). Omit $F_{\mathrm{o}}-F_{\mathrm{c}}$ electron density for the phosphate group is shown in light blue, contoured at $3 \sigma$. (d) Activity assay showing that the R1173A/R1260A double mutant is only mildly inhibited by activated SNF1. (e) Overlay of the structure of phosphorylated AC3-AC5 (in color) with that of AC3-AC5 in the holoenzyme (gray). Domains AC3-AC4 were used for the overlay, and the large conformational difference for domain AC5 corresponds to a rotation of $40^{\circ}$ and is indicated. (f) The position of Ser1157 moves by $16 \AA$ upon phosphorylation. Ser1157 interacts with different residues at the AC4-AC5 interface in the unphosphorylated ScACC holoenzyme structure. 
Table 1 Data collection and refinement statistics

\begin{tabular}{|c|c|}
\hline & Phosphorylated $A C 3-A C 5$ \\
\hline \multicolumn{2}{|l|}{ Data collection } \\
\hline Space group & $P 2_{1}$ \\
\hline \multicolumn{2}{|l|}{ Cell dimensions } \\
\hline$a, b, c(\AA)$ & $56.4,93.2,110.9$ \\
\hline$\alpha, \beta, \gamma\left(^{\circ}\right)$ & $90,99.6,90$ \\
\hline Resolution $(\AA)^{\mathrm{a}}$ & $50-2.9(3.00-2.9)$ \\
\hline$R_{\text {merge }}(\%)$ & $5.1(50.4)$ \\
\hline $\mathrm{CC}_{1 / 2}$ & $(0.798)$ \\
\hline$I / \sigma I$ & $19.2(2.3)$ \\
\hline Completeness ( $\%)$ & $98.3(99.2)$ \\
\hline Redundancy & $2.7(2.7)$ \\
\hline \multicolumn{2}{|l|}{ Refinement } \\
\hline Resolution (§) & $50-2.9$ \\
\hline No. of reflections & 25106 \\
\hline$R_{\text {work } /} R_{\text {free }}$ & $22.3 / 28.7$ \\
\hline \multicolumn{2}{|l|}{ No. of atoms } \\
\hline Protein & 6626 \\
\hline Ligand/ion & 2 \\
\hline Water & 0 \\
\hline \multicolumn{2}{|l|}{$B$-factors } \\
\hline Protein & 87.0 \\
\hline Ligand/ion & 73.9 \\
\hline Water & - \\
\hline \multicolumn{2}{|l|}{ R.m.s. deviations } \\
\hline Bond lengths (Å) & 0.010 \\
\hline Bond angles $\left({ }^{\circ}\right)$ & 1.3 \\
\hline
\end{tabular}

${ }^{a}$ One crystal was used for data collection. Highest resolution shell is shown in parenthesis.

compact shape for the holoenzyme (Figure 4b), using a buffer containing $10 \mathrm{~mm}$ Tris ( $\mathrm{pH} 7.5), 150 \mathrm{~mm} \mathrm{NaCl}$ and $100 \mathrm{~mm}$ sodium citrate to prepare the EM grids.

We selected particles from the images of the negatively stained specimens collected in the presence of citrate and carried out 2D class averaging. A total of 399 classes were obtained from 13129 particles (Supplementary Figure S5), many of them bearing resemblance to the crystal structure. In fact, the crystal structure of the holoenzyme could be readily overlaid onto these averages (Figure 4c), giving strong confirmation that we observed the same conformation of the holoenzyme by EM in this buffer condition.

Our EM observations suggest that the ScACC holoenzyme either assumes a defined, compact conformation or a continuum of extended conformations, depending on the buffer conditions. This conformational variability is important for the regulation of this enzyme (see next).

\section{Phosphorylation of Ser1157 stabilizes inactive ACC conformations}

With the establishment of a protocol to visualize the active conformation of ScACC by EM, we next assessed the effect of Ser1157 phosphorylation on the overall structure of the holoenzyme. We observed primarily elongated shapes for phosphorylated ScACC (Figure 4d), even in the presence of citrate, suggesting that phosphorylation of Ser1157 in ScACC inhibits the enzyme by promoting the dissociation of the $\mathrm{BC}$ domain dimer.

We proposed earlier that soraphen A inhibits eukaryotic ACC by binding to the $\mathrm{BC}$ domain and stabilizing the monomeric, inactive form of this domain in the holoenzyme [7, 19, 27]. We incubated the ScACC holoenzyme with soraphen A and then examined the sample by negative-stain EM in the presence of citrate. We again observed primarily elongated shapes in this sample (Figure 4e), providing direct experimental evidence in support of our model (Supplementary Figure S2).

\section{S1157A mutation has no obvious effect on yeast cell growth}

We next assessed the effect of ACC Ser1157 phosphorylation on yeast cell growth. We obtained haploid strains by sporulation of Saccharomyces cerevisiae strain W303D-ACC1 ${ }^{\Delta \text { Leu2 }}$ [28] transformed with plasmids carrying either wild-type (WT) $A C C 1$ or its S1157A mutant. Haploid strains in which one allele of $A C C 1$ was replaced by a LEU2 cassette could not survive on -LEU plate if no plasmid was complemented, confirming that $A C C 1$ is essential for survival (data not shown).

The haploid strains complemented with WT $A C C 1$ or its S1157A mutant were subjected to growth condition analysis. No significant difference was observed between the two strains when they are grown on glucose or sucrose, at either 30 or $20^{\circ} \mathrm{C}$ (Figure 5), suggesting that unregulated ScACC activity does not obviously affect cell growth under glucose-limitation conditions. Our observations are consistent with those from earlier studies on this mutant in yeast [22, 23]. Although mutation of Ser1157 did not produce an overall growth phenotype under the condition tested, it did lead to higher ACC activity, elevated fatty acid content and increased biosynthesis of other compounds derived from malonyl-CoA in yeast cells $[22,23]$. 
a

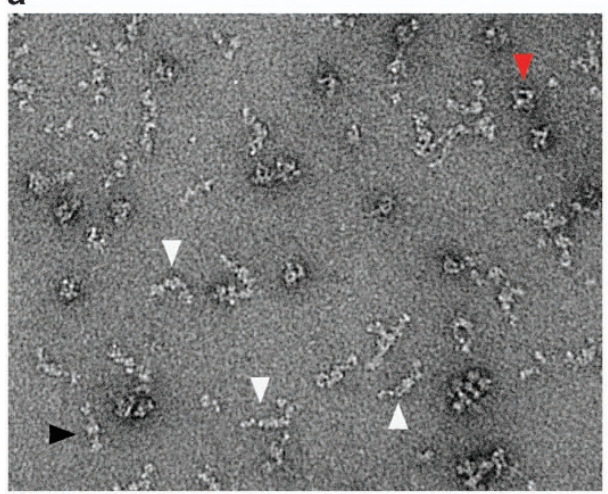

C

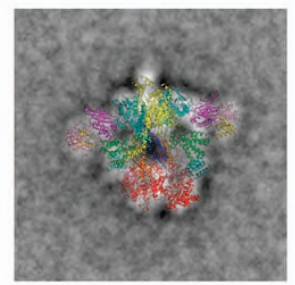

d

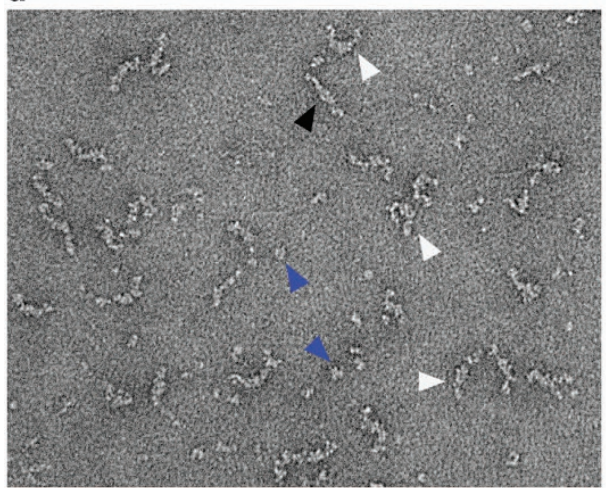

b

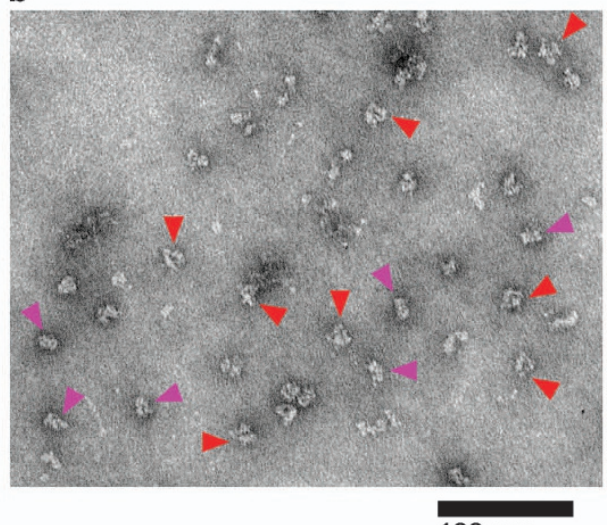

$100 \mathrm{~nm}$

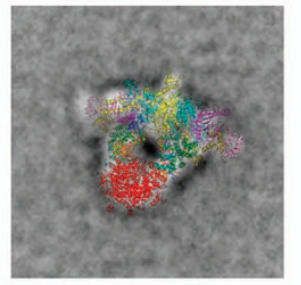

e

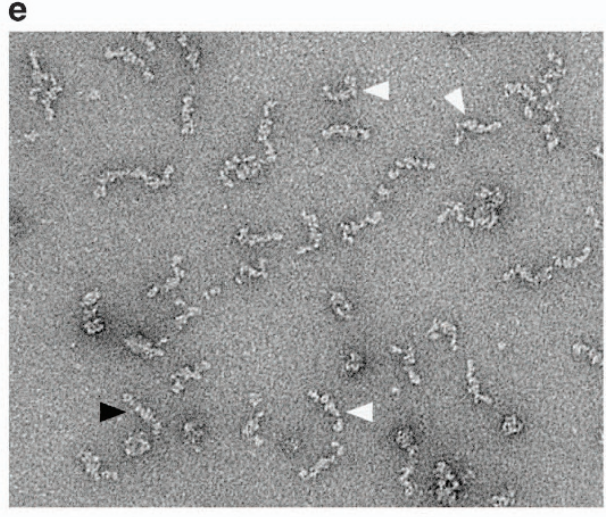

$100 \mathrm{~nm}$

Figure 4 Conformational variability of the yeast acetyl-CoA carboxylase (ScACC) holoenzyme dimer. (a) Electron microscopic (EM) image of negatively stained ScACC holoenzyme in the regular protein buffer $(20 \mathrm{~mm}$ Tris (pH 7.5) and $300 \mathrm{~mm} \mathrm{NaCl).}$ Predominantly elongated shapes were observed, both straight (black arrowhead) and bent (white arrowhead). A few compact shapes (red arrowhead) are likely similar to the structure observed in the crystal. (b) EM image of negatively stained ScACC holoenzyme in a buffer containing $10 \mathrm{~mm}$ Tris ( $\mathrm{pH}$ 7.5), $150 \mathrm{~mm} \mathrm{NaCl}$ and $100 \mathrm{~mm}$ sodium citrate. Mostly, compact shapes were observed, corresponding to front (red arrowhead) and side (magenta arrowhead) views of the structure observed in the crystal. (c) Three class averages of negatively stained ScACC in the presence of citrate. The crystal structure of ScACC was overlaid manually to indicate that the EM images are in good agreement with the crystal structure. (d) Negative-stain EM image of phosphorylated ScACC holoenzyme in a buffer containing $100 \mathrm{~mm}$ citrate. Predominantly elongated shapes were observed. Smaller particles (blue arrowhead) are likely the protein kinases used for phosphorylation (SNF1 and/or Tos3). (e) Negative-stain EM image of ScACC holoenzyme with soraphen A in a buffer containing $100 \mathrm{~mm}$ citrate. Predominantly elongated shapes were observed.

\section{A unified mechanism for ACC regulation by phosphorylation}

We proposed earlier a model for how phosphorylation of a Ser residue located before the $\mathrm{BC}$ domain core (Ser80 and Ser222 in human ACC1 and ACC2, respectively, Figure 1c) and soraphen $\mathrm{A}$ inhibit the catalytic activity of eukaryotic ACC [7] (Supplementary Figure S2). Essentially, phosphorylation and soraphen A stabilize a monomeric, catalytically inactive form of the BC domain, and our EM observations 


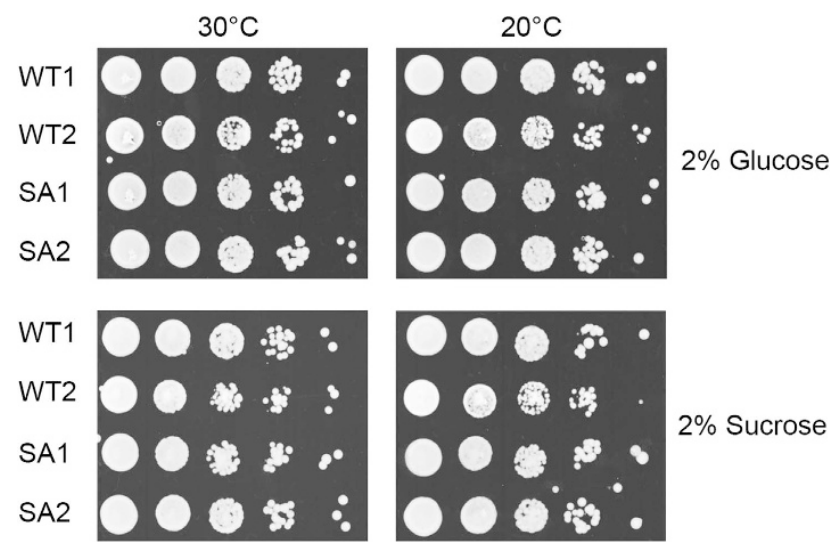

Figure 5 The S1157A mutant has no obvious effect on yeast cell growth under glucose-replete and glucose-limiting conditions. WT1 and WT2: two wild-type strains, SA1 and SA2: two S1157A mutant strains.

on ScACC with soraphen A provide direct evidence that the $\mathrm{BC}$ domain dimer has dissociated in the presence of this compound (Figure 4e). Moreover, when the $\mathrm{BC}$ domain is isolated away from the rest of the holoenzyme, it preferentially assumes the monomeric, inactive conformation [19].

Our EM studies indicate that phosphorylation at Ser1157 in the central region of ScACC also leads to the dissociation of the $\mathrm{BC}$ domain dimer. Therefore, we propose a unified molecular mechanism for how phosphorylation at both AMPK sites as well as soraphen A can allosterically inhibit ACC (Figure 6). The central feature of this mechanism is the dissociation of the $\mathrm{BC}$ domain dimer into inactive monomers. Once the $\mathrm{BC}$ dimer dissociates, the holoenzyme can assume a continuum of extended conformations, explaining the straight and bent shapes observed by EM (Figure 4d and e). This conformational dynamics is also consistent with observations on ACCs in an earlier study [24].

Ser1157 is $\sim 100 \AA$ from the BC domain dimer interface (Figure 1b). How does its phosphorylation lead to the dissociation of this dimer in the holoenzyme? We suggest that the conformational transition between AC3-AC5 domains in the holoenzyme and (phosphorylated) AC3-AC5 alone is the trigger (Figure 3e). The structure of AC3-AC5 alone represents the inactive conformation, which is stabilized by the phosphorylation of Ser1157. This explains why we did not observe any large changes in the structure of AC3-AC5 alone upon phosphorylation (Figure 3a). This situation is reminiscent of that for the $\mathrm{BC}$ domain, with the structure of $\mathrm{BC}$ domain alone being catalytically inactive and stabilized by phosphorylation on Ser80/Ser222 of human ACCs. On the other hand, phosphorylation of Ser1157 in the context of the holoenzyme would trigger a conformational change for domains $\mathrm{AC} 3-\mathrm{AC} 5$. The $40^{\circ}$ rotation of $\mathrm{AC} 3-\mathrm{AC} 4$ relative to AC5 (Figure 3e) is incompatible with the conformation of the holoenzyme observed in the crystal. It would lead to a displacement of the two BC domains in the holoenzyme relative to each other, which would disrupt the dimerization. The exact mechanism how the conformational change for domains $\mathrm{AC} 3-\mathrm{AC} 5$ is propagated to the $\mathrm{BC}$ domain dimer will await further studies.

\section{Discussion}

Citrate is a well-characterized activator of animal ACCs and is thought to function through promoting their polymerization into filaments [29]. On the other hand, citrate does not appear to have any effect on the catalytic activity of ScACC (Supplementary Figure S6). Although citrate is a part of the crystallization buffers for full-length ScACC and many of its domains, we have so far failed to find any ordered citrate molecule in our structures. However, our EM studies clearly show that citrate has an impact on the overall structure of ScACC. Citrate may thus have an indirect, non-specific effect by stabilizing the overall structure of the holoenzyme.

We observed the active conformation of ScACC by EM using a buffer solution derived from the crystallization condition. The protocol of using crystallization screening to identify suitable conditions for EM studies could have wider applicability. A sample of interest could be screened (robotically) against the large collection of crystallization buffers currently available, and conditions that give (micro)crystals could then be selected for EM studies.

The ACC CT domain dimer has an extensive interface [30] and represents an anchor in the ACC holoenzyme dimer. On the other hand, the $\mathrm{BC}$ domain dimer appears to be a weak link in the ACC holoenzyme dimer. The domain readily distributes between the active, dimeric form (where the holoenzyme would be more compact) and the inactive, monomeric forms (leading to a continuum of elongated conformations of the holoenzyme). Citrate can affect the equilibrium between the two states. It is likely that in cells the compact, active conformation of ACC dominates owing to the various compounds present in the cytosol. The elongated, inactive conformations are observed with our purified, unphosphorylated 

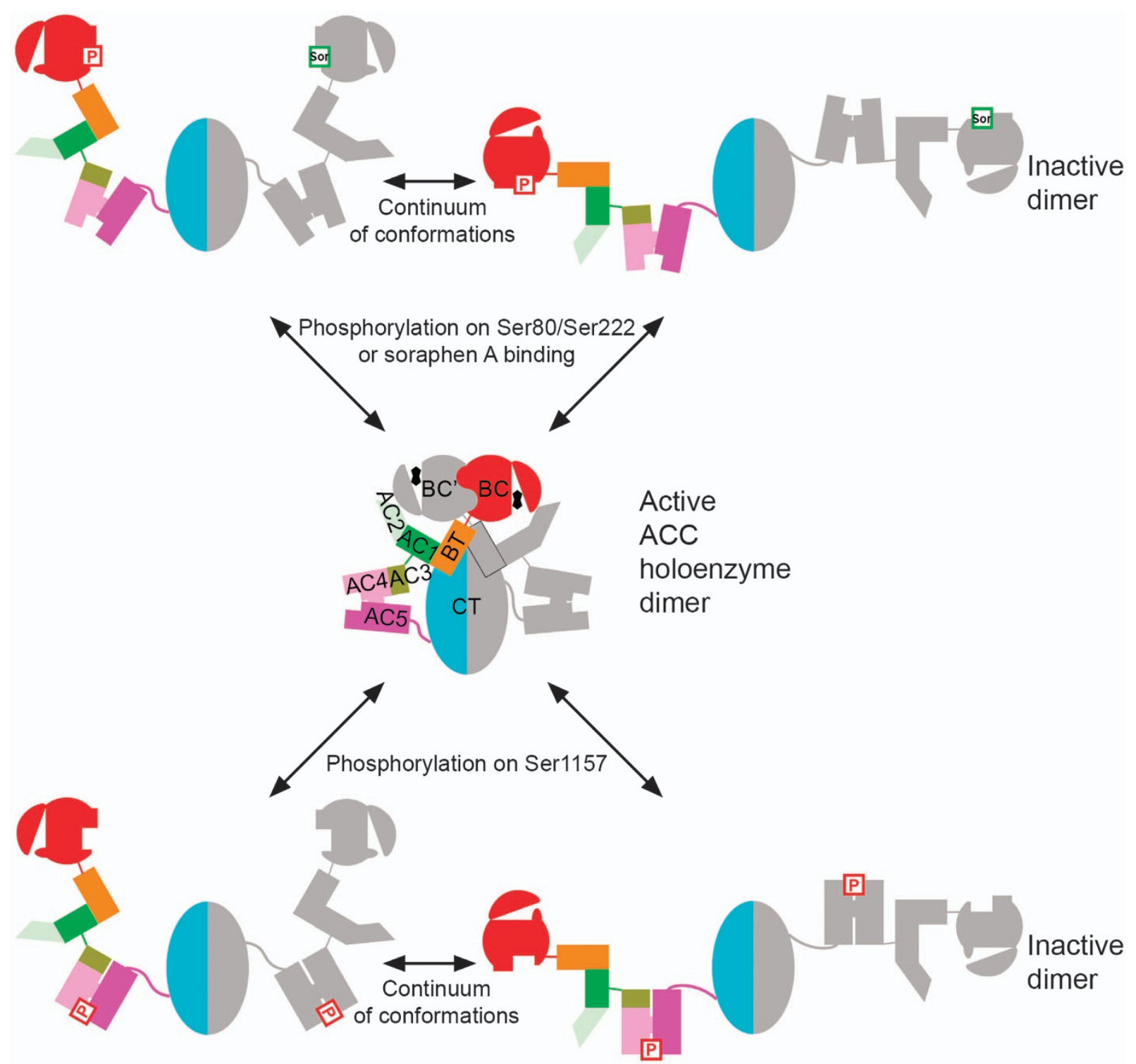

Bent shape

Straight shape

Figure 6 A unified molecular mechanism for the inhibition of eukaryotic acetyl-CoA carboxylases (ACCs) by phosphorylation and soraphen A binding. Phosphorylation at the site before the biotin carboxylase (BC) domain core in animal ACCs (indicated with Ser80 and Ser222 in human ACC1 and ACC2, respectively, labeled P), soraphen A binding (labeled Sor) and phosphorylation in the central region (indicated with Ser1157 in yeast ACC (ScACC)) all stabilize the monomeric form of the BC domain. The monomeric $\mathrm{BC}$ domain has large conformational changes in the dimer interface and in the active site region, which blocks biotin binding and thereby catalysis. Biotin is indicated with the fused pentagons in black. Once the BC domain dimer dissociates, the holoenzyme can assume a continuum of elongated conformations, from bent to straight shapes, as observed by electron microscope.

sample probably because the enzyme was placed in a buffer that is too distinct from the physiological condition.

The structure of CT domain alone is essentially the same as that in the holoenzyme. In contrast, the structure of BC domain or AC3-AC5 domains alone shows extensive differences to that in the holoenzyme. The conformational flexibility is important for the regulation of this enzyme. On the other hand, this also serves as a cautionary tale for the 'divide-and-conquer' approach, in that the structures of the isolated domains may not always recapitulate the situation in the context 
of the full-length protein. At the same time, any conformational differences that are observed may have important roles in the functions of the full-length protein.

Soraphen A inhibits eukaryotic ACCs by taking advantage of their conformational variability. A class of highly potent inhibitors of human ACCs was recently developed based on the soraphen A-binding site [31], indicating a new approach for developing ACC inhibitors. It might be possible that there are other mechanisms of stabilizing the elongated conformations of the holoenzyme. Such compounds would be inhibitors of ACC and could be promising leads for drug discovery as well.

\section{Materials and Methods}

\section{Protein expression and purification}

Residues 22-2 233 and domains AC3-AC5 of ScACC (residues $1036-1503$ ) were overexpressed at $25^{\circ} \mathrm{C}$ in E. coli BL21(DE3) Rosetta cells and purified as described previously [7], with a modification that $2 \mathrm{~mm}$ dithiothreitol was included in the gel filtration buffer.

The three subunits of S. cerevisiae SNF1 (residues 41-633 of Snf1, 250-418 of Gal83 and 1-322 of Snf4) were overexpressed together, using a polycistronic plasmid built from pET28a (Novagen, Madison, WI, USA) [25], in E. coli BL21(DE3) Star cells at $25^{\circ} \mathrm{C}$. Gal 83 carried a hexa-His tag. Cells were lysed by sonication in a buffer containing $20 \mathrm{~mm}$ Tris ( $\mathrm{pH} 7.5), 150 \mathrm{~mm}$ $\mathrm{NaCl}, 5 \%$ (v/v) glycerol, $0.1 \%$ (v/v) Triton X-100 and 10 mм $\beta$ mercaptoethanol. The complex was purified by Ni-NTA (Qiagen, Hilden, Germany) and gel filtration chromatography (Sephacryl S-300, GE Healthcare, Pittsburgh, PA, USA) in a buffer containing $20 \mathrm{~mm}$ Tris ( $\mathrm{pH} 7.5$ ), $150 \mathrm{~mm} \mathrm{NaCl}$ and $2 \mathrm{~mm}$ dithiothreitol.

The segment containing residues 11-460 of S. cerevisiae Tos3 was inserted into pET26b (Novagen) and overexpressed in E. coli BL21(DE3) Star cells at $25^{\circ} \mathrm{C}$. The recombinant protein with a C-terminal hexa-His tag was purified following the same procotol as that for the SNF1 complex.

\section{Mutagenesis}

Site-specific and deletion mutations were introduced with the QuikChange Kit (Agilent, Santa Clara, CA, USA) and sequenced for confirmation. In deletion mutant $\Delta 1137-1170$, residues $1137-1170$ in domain AC4 were replaced by a (Gly) linker.

\section{In vitro phosphorylation, activity and gel shift assays}

The in vitro phosphorylation reactions contained $4 \mu \mathrm{M}$ ACC, $0.8 \mu \mathrm{m}$ SNF1, $0.2 \mu \mathrm{m}$ Tos3, $2 \mathrm{~mm}$ ATP and $5 \mathrm{~mm} \mathrm{MgCl}_{2}$. The reaction was carried out at room temperature. Incubation at higher temperature $\left(37^{\circ} \mathrm{C}\right)$ or with higher concentration of ATP would induce precipitation.

The catalytic activity of ACC was determined using a coupled enzyme assay, converting the hydrolysis of ATP to the disappearance of NADH [32]. The reaction mixture contained $100 \mathrm{~mm}$ HEPES (pH 7.5), $8 \mathrm{~mm} \mathrm{MgCl}_{2}, 40 \mathrm{~mm} \mathrm{KHCO}_{3}$, $200 \mathrm{~mm} \mathrm{KCl}, 0.2 \mathrm{~mm} \mathrm{NADH}, 0.5 \mathrm{~mm}$ phosphoenolpyruvate, $0.5 \mathrm{~mm}$ ATP, 6 units of lactate dehydrogenase (Sigma, St Louis, MO, USA), 4 units of pyruvate kinase, $100 \mathrm{~nm} \mathrm{ACC} \mathrm{and} 0.1$ or $1 \mathrm{~mm}$ acetyl-CoA. The absorbance at $340 \mathrm{~nm}$ was monitored for $60 \mathrm{~s}$.

For gel shift assays, domains AC3-AC5 of yeast ACC $(40 \mu \mathrm{M})$ were incubated with $1 \mu \mathrm{M}$ SNF1, $0.2 \mu \mathrm{M}$ Tos3, $0.75 \mathrm{~mm}$ ATP and $0.75 \mathrm{mM} \mathrm{MgCl}_{2}$ for $20 \mathrm{~min}$ at room temperature before being separated by sodium dodecyl sulfate polyacrylamide gel electrophoresis. Reactions lacking SNF1 and/or Tos3 were included as controls.

\section{Protein crystallization}

Domains AC3-AC5 were incubated in the in vitro phosphorylation system with a buffer of $20 \mathrm{~mm}$ Tris ( $\mathrm{pH} 7.5), 450 \mathrm{~mm}$ $\mathrm{NaCl}, 2 \mathrm{~mm}$ ATP and $5 \mathrm{~mm} \mathrm{MgCl} 2$ at $20^{\circ} \mathrm{C}$ for $20 \mathrm{~min}$ before crystallization. The final concentration of AC3-AC5 was $2.9 \mathrm{mg} \mathrm{ml}^{-1}$ and the molar ratio of AC3-AC5:SNF1:Tos3 was 225:5:1. Crystals of phosphorylated AC3-AC5 were obtained at $20^{\circ} \mathrm{C}$ using the sitting-drop vapor diffusion method. The precipitant solution contained $100 \mathrm{~mm}$ HEPES (pH 7.5), $3 \%(\mathrm{v} / \mathrm{v})$ MPD, $2.5 \mathrm{~mm}$ sodium citrate and 5\% (v/v) glycerol. The crystals appeared within several minutes of setup and were harvested after 2 days. Glycerol was used as the cryo-protectant and crystals were flash frozen in liquid nitrogen for data collection at $100 \mathrm{~K}$.

\section{Data collection and structure determination}

An X-ray diffraction data set of phosphorylated AC3-AC5 domains was collected to $2.9 \AA$ A resolution at NE-CAT beamline 24ID-E of Advanced Photon Source, with an ADSC Q315 charge-coupled device detector (Poway, CA, USA). The diffraction images were processed with the HKL program [33]. The crystal belonged to space group $P 2_{1}$, with cell parameters of $a=56.4 \AA, b=93.2 \AA, c=110.9 \AA$ and $\beta=99.6^{\circ}$. There are two molecules in the asymmetric unit, both containing phosphorylated Ser1157. The structure of unphosphorylated AC3-AC5 domains was used as the search model to solve the structure by molecular replacement with the program Phaser [34]. The final atomic model was built with Coot [35] and refined with PHENIX [36].

\section{EM and image processing}

ScACC and phosphorylated ScACC were diluted to $0.05 \mathrm{mg} \mathrm{ml}^{-1}$ concentration in buffer A (20 mM Tris ( $\left.\mathrm{pH} 7.5\right)$, and $300 \mathrm{~mm} \mathrm{NaCl}$ ) for initial EM studies. Subsequently, samples were incubated overnight in buffer B (10 mM Tris ( $\mathrm{pH} 7.5)$, $150 \mathrm{~mm} \mathrm{NaCl}$ and $100 \mathrm{~mm}$ sodium citrate) and diluted to $0.05 \mathrm{mg} \mathrm{ml}^{-1}$ in $200 \mathrm{~mm}$ sodium citrate. To study the effect of soraphen A binding, ScACC was incubated with $100 \mu \mathrm{m}$ Soraphen A overnight in buffer B and diluted to $0.05 \mathrm{mg} \mathrm{ml}^{-1}$ in $200 \mathrm{~mm}$ sodium citrate.

Protein samples were prepared for EM by conventional negative staining with $0.7 \%(\mathrm{w} / \mathrm{v})$ uranyl formate. The negatively stained images were collected at room temperature with a Philips CM10 electron microscope (FEI, Hillsboro, OR, USA) 
equipped with a tungsten filament and operated at $100 \mathrm{kV}$. Images were recorded on an AMT XR16L-ActiveVu chargecoupled device camera (Woburn, MA, USA) using a defocus of $\sim 1.5 \mu \mathrm{m}$ and a nominal magnification of $\times 50000$.

A total of 13129 particles were picked manually from 51 charge-coupled device images and windowed into $112 \times 112$ pixel images with program e2boxer.py of the EMAN2 software package [37]. After reduction of the particle images to $64 \times 64$ pixels, the particles were centered, aligned to each other and classified with the iterative stable alignment and clustering (ISAC) [38] procedure implemented in the SPARX software package [39], specifying 50 images per group and a pixel error threshold of 0.7. After 15 generations of iterative stable alignment and clustering, 399 classes were obtained, accounting for 8 896 particles $(67.8 \%$ of the entire data set). Averages of these classes were calculated using the original $112 \times 112$-pixel images.

\section{Yeast strains, media and growth conditions}

The $S$. cerevisiae diploid strain W303D-ACC1 $1^{\Delta \text { Leu2 }}$ and pRS426 plasmid carrying the yeast $A C C 1$ gene were generous gifts from Dr P Gornicki at the University of Chicago [28]. The $A C C 1$ gene together with its upstream promoter was amplified by PCR from the pRS426 plasmid and inserted into pRS416 vector using the restriction enzymes SacI and XhoI (New England Biolabs, Ipswich, MA, USA).

Rich medium (yeast extract-peptone-dextrose), synthetic complete medium, sporulation medium and genetic methods were as described [40]. The yeast strains used here are listed in Supplementary Table S1. Both WT and S1157A strains were haploid strains obtained by sporulation of W303D-ACC1 $1^{\Delta \text { Leu2 }}$ transformed with pRS416 plasmids carrying either WT or S1157A mutant $A C C 1$ gene.

For growth condition assays, serial dilutions of log-phase cultures in synthetic complete-Ura medium were spotted on synthetic complete-Ura or sucrose-Ura $(0.67 \%$ yeast nitrogen base, $2 \%$ sucrose, $1.92 \mathrm{~g} \mathrm{l}^{-1}$ yeast synthetic drop-out medium supplements without urail, $2 \%$ agar) plates for 2 days at $30{ }^{\circ} \mathrm{C}$ or for 5 days at $20^{\circ} \mathrm{C}$.

\section{Conflict of Interest}

The authors declare no conflict of interest.

\section{Acknowledgements}

We thank P Gornicki for the yeast ACC plasmid and S Banerjee, K Perry, R Rajashankar, J Schuermann and N Sukumar for access to NE-CAT 24-C and 24-E beamlines at the Advanced Photon Source. This research was supported by NIH grants R01DK067238 and S10OD012018 (to LT) and R01GM041784 (to LSS). This work is based upon research conducted at the Northeastern Collaborative Access Team beamlines, which are funded by the National Institute of General Medical Sciences from the National Institutes of Health (P41 GM103403). The Pilatus 6M detector on 24-ID-C beam line is funded by a NIH-ORIP HEI grant (S10 RR029205). This research used resources of the Advanced
Photon Source, a US Department of Energy (DOE) Office of Science User Facility operated for the DOE Office of Science by Argonne National Laboratory under Contract No. DE-AC02-06CH11357.

\section{Author contributions}

JW carried out protein expression, purification, crystallization, diffraction data collection and structure determination, enzymatic assays, site-directed mutagenesis and gel shift assay. JW and YZ carried out EM analysis. JW and TYY carried out yeast studies. GAA and MJR produced the SNF1 expression plasmid. KS produced the Tos3 expression plasmid and set up the in vitro phosphorylation system monitored by gel shift, and JW improved it for activity assays and crystallization. LT, TW and LSS analyzed the data and supervised the research. JW and LT wrote the paper with inputs from all authors.

\section{References}

1 Tong L. Structure and function of biotin-dependent carboxylases. Cell Mol Life Sci 2013; 70: 863-891.

2 Waldrop GL, Holden HM, St. Maurice M. The enzymes of biotin dependent $\mathrm{CO} 2$ metabolism: what structures reveal about their reaction mechanisms. Prot Sci 2012; 21: 1597-1619.

3 Cronan JE Jr., Waldrop GL. Multi-subunit acetyl-CoA carboxylases. Prog Lipid Res 2002; 41: 407-435.

4 Polyak SW, Abell AD, Wilce MCJ, Zhang L, Booker GW. Structure, function and selective inhibition of bacterial acetyl-CoA carboxylase. Appl Microbiol Biotechnol 2012; 93: 983-992.

5 Abramson HN. The lipogenesis pathway as a cancer target. J Med Chem 2011; 54: 5615-5638.

6 Wakil SJ, Abu-Elheiga LA. Fatty acid metabolism: target for metabolic syndrome. J Lipid Res 2009; 50: S138-S143.

7 Wei J, Tong L. Crystal structure of the 500-kDa yeast acetyl-CoA carboxylase holoenzyme dimer. Nature 2015; 526: 723-727.

8 St. Maurice M, Reinhardt L, Surinya KH et al. Domain architecture of pyruvate carboxylase, a biotin-dependent multifunctional enzyme. Science 2007; 317: 1076-1079.

9 Xiang S, Tong L. Crystal structures of human and Staphylococcus aureus pyruvate carboxylase and molecular insights into the carboxyltransfer reaction. Nat Struct Mol Biol 2008; 15: 295-302.

10 Huang CS, Sadre-Bazzaz K, Shen Y, Deng B, Zhou ZH, Tong L. Crystal structure of the a6b6 holoenzyme of propionyl-coenzyme A carboxylase. Nature 2010; 466: 1001-1005.

11 Huang CS, Ge P, Zhou ZH, Tong L. An unanticipated architecture of the $750-\mathrm{kDa}$ a6b6 holoezyme of 3-methylcrotonyl-CoA carboxylase. Nature 2012; 481: 219-223.

12 Fan C, Chou C-Y, Tong L, Xiang S. Crystal structure of urea carboxylase provides insights into the carboxyltransfer reaction. J Biol Chem 2012; 287: 9389-9398. 
13 Tran TH, Hsiao Y-S, Jo J et al. Structure and function of a single-chain, multi-domain long-chain acyl-CoA carboxylase. Nature 2015; 518: 120-124.

14 Jurado AR, Huang CS, Zhang X, Zhou ZH, Tong L. Structure and substrate selectivity of the $750-\mathrm{kDa}$ a6b6 holoenzyme of geranyl-CoA carboxylase. Nat Commun 2015; 6: 8986.

15 Hardie DG, Schaffer BE, Brunet A. AMPK: an energysensing pathway with multiple inputs and outputs. Trends Cell Biol 2016; 26: 190-201.

16 Carling D, Viollet B. Beyond energy homeostasis: the expanding role of AMP-activated protein kinase in regulating metabolism. Cell Metab 2015; 21: 799-804.

17 Hedbacker K, Carlson M. SNF1/AMPK pathways in yeast. Front Biosci 2008; 13: 2408-2420.

18 Cho YS, Lee JI, Shin D et al. Molecular mechanism for the regulation of human ACC2 through phosphorylation by AMPK. Biochem Biophys Res Commun 2010; 391: 187-192.

19 Shen Y, Volrath SL, Weatherly SC, Elich TD, Tong L. A mechanism for the potent inhibition of eukaryotic acetylcoenzyme A carboxylase by soraphen A, a macrocyclic polyketide natural product. Mol Cell 2004; 16: 881-891.

20 Gruhler A, Olsen JV, Mohammed S et al. Quantitative phosphoproteomics applied to the yeast pheromone signaling pathway. Mol Cell Proteomics 2005; 4: 310-327.

21 Holt LJ, Tuch BB, Villen J, Johnson AD, Gygi SP, Morgan DO. Global analysis of Cdk1 substrate phosphorylation sites provides insights into evolution. Science 2009; 325: 1682-1686.

22 Shi S, Chen Y, Siewers V, Nielsen J. Improving production of malonyl coenzyme A-derived metabolites by abolishing Snfl-dependent regulation of Acc1. mBio 2014; 5: e01130-e01114.

23 Choi JW, Da Silva NA. Improving polyketide and fatty acid synthesis by engineering of the yeast acetyl-CoA carboxylase. J Biotech 2014; 187: 56-59.

24 Hunkeler M, Stuttfeld E, Hagmann A, Imseng S, Maier T. The dynamic organization of fungal acetyl-CoA carboxylase. Nat Commun 2016; 7: 11196.

25 Amodeo GA, Rudolph MJ, Tong L. Crystal structure of the heterotrimer core of Sacharyomyces cerevisiae AMPK homolog SNF1. Nature 2007; 449: 492-495.

26 Hong S-P, Leiper FC, Woods A, Carling D, Carlson M. Activation of yeast Snfl and mammalian AMP-activated protein kinase by upstream kinases. Proc Natl Acad Sci USA 2003; 100: 8839-8843.

27 Shen Y, Chou C-Y, Chang G-G, Tong L. Is dimerization required for the catalytic activity of bacterial biotin carboxylase? Mol Cell 2006; 22: 807-818.

28 Joachimiak M, Tevzadze G, Podkowinski J, Haselkorn R, Gornicki P. Wheat cytosolic acetyl-CoA carboxylase complements an ACC1 null mutation in yeast. Proc Natl Acad Sci USA 1997; 94: 9990-9995.
29 Kleinschmidt AK, Moss J, Lane MD. Acetyl coenzyme A carboxylase: filamentous nature of the animal enzymes. Science 1969; 166: 1276.

30 Zhang H, Yang Z, Shen Y, Tong L. Crystal structure of the carboxyltransferase domain of acetyl-coenzyme A carboxylase. Science 2003; 299: 2064-2067.

31 Harriman G, Greenwood J, Bhat S et al. Acetyl-CoA carboxylase inhibition by ND-630 reduces hepatic steatosis, improves insulin sensitivity, and modulates dyslipidemia in rats. Proc Natl Acad Sci USA 2016; 113: E1796-E1805.

32 Blanchard CZ, Lee YM, Frantom PA, Waldrop GL. Mutations at four active site residues of biotin carboxylase abolish substrate-induced synergism by biotin. Biochem 1999; 38: 3393-3400.

33 Otwinowski Z, Minor W. Processing of X-ray diffraction data collected in oscillation mode. Method Enzymol 1997; 276: 307-326.

34 McCoy AJ, Grosse-Kunstleve RW, Adams PD, Winn MD, Storoni LC, Read RJ. Phaser crystallographic software. J Appl Cryst 2007; 40: 658-674.

35 Emsley P, Cowtan KD. Coot: model-building tools for molecular graphics. Acta Cryst 2004; D60: 2126-2132.

36 Adams PD, Grosse-Kunstleve RW, Hung L-W et al. PHENIX: building a new software for automated crystallographic structure determination. Acta Cryst 2002; D58: 1948-1954.

37 Ludtke SJ, Baldwin PR, Chiu W. EMAN: semiautomated software for high-resolution single-particle reconstructions. J Struct Biol 1999; 128: 82-97.

38 Yang Z, Fang J, Chittuluru J, Asturias FJ, Penczek PA. Iterative stable alignment and clustering of 2D transmission electron microscope images. Structure 2012; 20: 237-247.

39 Hohn M, Tang G, Goodyear G et al. SPARX, a new environment for Cryo-EM image processing. J Struct Biol 2007; 157: 47-55.

40 Amberg DC, Burke DJ, Strathern JN. Methods in yeast genetics: A Cold Spring Harbor Laboratory Course Manual. Cold Spring Harbor Lab Press: Plainview, NY, USA. 2005.

(Supplementary information is linked to the online version of the paper on the Cell Discovery website.)

(i) This work is licensed under a Creative Commons Attribution 4.0 International License. The images or other third party material in this article are included in the article's Creative Commons license, unless indicated otherwise in the credit line; if the material is not included under the Creative Commons license, users will need to obtain permission from the license holder to reproduce the material. To view a copy of this license, visit http://creativecommons.org/licenses/by/4.0/

(C) The Author(s) 2016 